\title{
Time dependent CPV in the beauty sector
}

\author{
Sean Benson \\ on behalf of the $\mathrm{LHCb}, \mathrm{BaBar}$, and Belle collaborations
}

Time-dependent $C P$ violating phenomena probe the time evolution and the decay of beauty hadrons and allow access to the underlying dynamics. We present various time-dependent $C P$ violation measurements in the $B^{0}$ and $B_{\mathrm{s}}^{0}$ meson systems that are performed using $p p$ collisions by $\mathrm{LHCb}$ and using $e^{+} e^{-}$collisions by the $\mathrm{B}$ factory experiments $\mathrm{BaBar}$ and Belle. For $\mathrm{LHCb}$, we report measurements of the quantumloop mediated $B_{\mathrm{s}}^{0} \rightarrow\left(\mathrm{K}^{-} \pi^{+}\right)\left(\mathrm{K}^{+} \pi^{-}\right)$and $B_{\mathrm{s}}^{0} \rightarrow \phi \phi$ decays, and measurements of $B^{0} \rightarrow D^{\mp} \pi^{ \pm}$and $B_{\mathrm{s}}^{0} \rightarrow h^{+} h^{-}$decays. For Belle, we report new results for the charmless $B^{0} \rightarrow \pi^{0} \pi^{0} \mathrm{~K}_{S}^{0}$ and $B^{0} \rightarrow \mathrm{K}_{S}^{0} \eta \gamma$ decays. In addition, we present a measurement of $\cos 2 \beta$ obtained by a time-dependent Dalitz plot analysis of $B^{0} \rightarrow D^{*} h^{0}$ with $D \rightarrow \mathrm{K}_{S}^{0} \pi^{+} \pi^{-}$that combines the final BaBar and Belle data sets.

\section{Introduction}

Measurements of $C P$ asymmetries in neutral $B$ mesons probe the interference of the mixing and decay Feynman diagrams. The $C P$ asymmetry as a function of time can be described as

$$
A_{C P}=\frac{\Gamma_{\left.\bar{B}_{(} d, s\right)^{0} \rightarrow f}-\Gamma_{B_{(}(d, s)^{0} \rightarrow f}}{\Gamma_{\bar{B}(d, s)^{0} \rightarrow f}+\Gamma_{B_{(d, s)^{0} \rightarrow f}}}=\frac{-C_{f} \cos \left(\Delta m_{d, s} t\right)+S_{f} \sin \left(\Delta m_{d, s} t\right)}{\cosh \left(\Delta \Gamma_{d, s} t / 2\right)+A_{f}^{\Delta \Gamma} \sinh \left(\Delta \Gamma_{d, s} t / 2\right)},
$$

where

$$
C_{f} \equiv \frac{1-\left|\lambda_{f}\right|}{1+\left|\lambda_{f}\right|}, C_{f} \equiv \frac{2 I m \lambda_{f}}{1+\left|\lambda_{f}\right|}, A_{f}^{\Delta \Gamma} \equiv-\frac{2 R e \lambda_{f}}{1+\left|\lambda_{f}\right|}, \lambda_{f} \equiv \frac{q}{p} \frac{\bar{A}_{f}}{A_{f}} .
$$

In the above equation, $f$ represents the final state, $A_{f}$ the $B_{s, d}$ decay amplitude, and $\bar{A}_{f}$ the $\bar{B}_{s, d}$ decay amplitude.

In the Standard Model (SM), the weak phase from $B_{\mathrm{s}}^{0}-\bar{B}_{\mathrm{s}}^{0}$ mixing is given by $\phi_{\mathrm{s}}^{c \overline{c s}} \approx$ $-2 \beta_{s}=2 \arg \left(-V_{t s} V_{t b}^{*} / V_{c s} V_{c b}^{*}\right)$. The SM prediction for $\phi_{\mathrm{s}}^{c \overline{c s}}$ has been obtained from global fits to experimental data yielding a value of $-0.0360 \pm 0.0006 \mathrm{rad}$ [1]. There are however beyond-the-SM theories that could provide additional contributions to $B_{\mathrm{s}}^{0}$ mixing diagrams which alter this value $[2,3]$.

In addition to decays with a tree-level contribution, measurements of decays in which the leading order contribution is a flavour changing neutral current (FCNC)

Nikhef National Institute for Subatomic Physics

Amsterdam, Netherlands, e-mail: sean.benson@cern.ch 
interaction are important in the search for physics beyond the SM. The $C P$-violating phase measured in the $B_{\mathrm{s}}^{0} \rightarrow \phi \phi$ decay results from $\mathrm{b} \rightarrow \mathrm{s} \overline{\mathrm{s} s}$ transitions and is therefore expected to be close to zero in the SM due to the effective cancellation of the $C P$-violating weak phase between the $B_{\mathrm{s}}^{0}$ mixing diagrams and the penguin decay diagrams $[5,6]$. The $B_{\mathrm{s}}^{0} \rightarrow \mathrm{K}^{* 0} \overline{\mathrm{K}}^{* 0}(892)$ decay is an example of a $\mathrm{b} \rightarrow d \bar{d} \mathrm{~s}$ transition and the associated $C P$-violating phase is expected to be zero for similar reasons as the $B_{\mathrm{s}}^{0} \rightarrow \phi \phi$ decay.

Time-dependent $C P$ violation measurements in the $B^{0}$ system are mainly performed using b-factory data. The high flavour tagging efficiency of the b-factories makes many analyses of decay channels with event yields of as little as a hundred events possible. For time-dependent analyses in the $B^{0}$ system at the b-factories, the decay time difference with respect to the other $\bar{B}^{0}$ decay produced in the event is measured and denoted as $\Delta t$. The time-dependent decay rate is then of the form

$$
\mathcal{P}(\Delta t)=\frac{e^{-|\Delta t| / \tau_{B^{0}}}}{4 \tau_{B^{0}}}\left\{1+q\left[\mathcal{S} \sin \left(\Delta m_{d} \Delta t\right)+\mathcal{A} \cos \left(\Delta m_{d} \Delta t\right)\right]\right\},
$$

where $q$ is the $B^{0}$ meson flavour, $\mathcal{S}$ and $\mathcal{A}$ are the $S_{f}$ and $C_{f}$ parameters in eq. 1 , respectively. Time-dependent analyses in the $B^{0}$ system are sensitive to the CKM angle $\beta \equiv \phi_{1}$, the current central value of $\sin (2 \beta)$ is $-0.699 \pm 0.017$ [4]. Measurements of $\sin (2 \beta)$ result in an ambiguity in the sign of $\cos (2 \beta)$.

The following sections summarise updated and most accurate measurements of the $C P$-violating weak phases in $\mathrm{b} \rightarrow c \bar{c} \mathrm{~s}$ and $\mathrm{b} \rightarrow \bar{s} \bar{s}$ transitions $[9,10,11]$, in addition to a first measurement of the weak phase in $\mathrm{b} \rightarrow d \bar{d}$ s transitions from $\mathrm{LHCb}$ data [11]. Decay time dependent measurements of $B^{0} \rightarrow \pi^{0} \pi^{0} \mathrm{~K}_{S}^{0}$ and $B^{0} \rightarrow \mathrm{K}_{S}^{0} \eta \gamma$ decays are presented using Belle data [12,13], along with a new measurement of $\cos (2 \beta)$ using a combination of BaBar and Belle data that provides first evidence that $\cos (2 \beta)>0[14]$.

\section{Time-dependent $C P$ violation in $B_{\mathrm{s}}^{0}$ decays}

\subsection{The $B_{\mathrm{s}}^{0} \rightarrow \mathrm{J} / \psi \mathrm{K}^{+} \mathrm{K}^{-}$decay}

The $B_{\mathrm{s}}^{0} \rightarrow \mathrm{J} / \psi \mathrm{K}^{+} \mathrm{K}^{-}$decay requires the use of decay angles in the helicity basis to disentangle the four polarisation amplitudes contributing to the decay ( $3 P$-wave and an $S$-wave). The four observables are shown in Figure 1.

A good understanding of the efficiencies as a function of each observable is important in keeping measurements dominated by statistical uncertainties. The measurement uses 95690 candidates obtained from $3 \mathrm{fb}^{-1}$ of LHCb data collected in 2011 and 2012 [9]. The decay gives access to the $B_{\mathrm{s}}^{0}-\bar{B}_{\mathrm{s}}^{0}$ oscillation frequency, $\Delta m_{\mathrm{s}}$, the decay width and decay width difference, $\Gamma_{\mathrm{s}} \& \Delta \Gamma_{\mathrm{s}}$, in addition to the decay amplitudes, $\left|A_{0}\right|^{2} \&\left|A_{\perp}\right|^{2}$, and the $C P$-violating parameters, $\phi_{\mathrm{s}}^{c \overline{c s}} \&|\lambda|$. The results of 

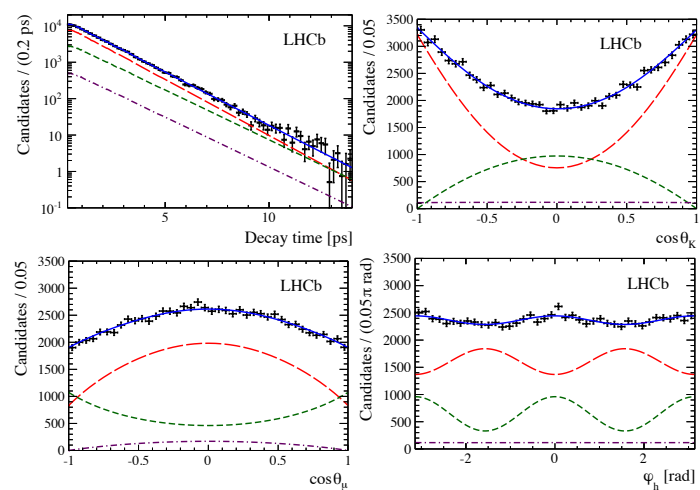

Fig. 1 Decay-time and helicity-angle distributions for $B_{\mathrm{s}}^{0} \rightarrow \mathrm{J} / \psi \mathrm{K}^{+} \mathrm{K}^{-}$decays (data points) with the one-dimensional fit projections overlaid. The solid blue line shows the total signal contribution, which is composed of $C P$-even (long-dashed red), $C P$-odd (short-dashed green) and $\mathrm{S}$-wave (dotted-dashed purple) contributions [9].

the measurement are provided in Table 1. Results are consistent with SM predictions and result in stringent constraints on physics beyond the SM [15].

Table 1 Values of the principal physics parameters determined from the polarisation-independent fit. The first uncertainty is statistical and the second systematic.

\begin{tabular}{lc} 
Parameter & Value \\
\hline$\Gamma_{\mathrm{s}}\left[\mathrm{ps}^{-1}\right]$ & $0.6603 \pm 0.0027 \pm 0.0015$ \\
$\Delta \Gamma_{\mathrm{s}}\left[\mathrm{ps}^{-1}\right]$ & $0.0805 \pm 0.0091 \pm 0.0032$ \\
$\left|A_{\perp}\right|^{2}$ & $0.2504 \pm 0.0049 \pm 0.0036$ \\
$\left|A_{0}\right|^{2}$ & $0.5241 \pm 0.0034 \pm 0.0067$ \\
$\phi_{\mathrm{s}}^{c \overline{c s}}[\mathrm{rad}]$ & $-0.058 \pm 0.049 \pm 0.006$ \\
$|\lambda|$ & $0.964 \pm 0.019 \pm 0.007$ \\
$\Delta m_{\mathrm{S}}\left[\mathrm{ps}^{-1}\right]$ & $17.711_{-0.057}^{+0.055} \pm 0.011$ \\
\hline
\end{tabular}

\subsection{The $B_{\mathrm{s}}^{0} \rightarrow \phi \phi$ decay}

The $B_{\mathrm{s}}^{0} \rightarrow \phi \phi$ decay requires an angular analysis to disentangle the polarisation states, as for the $B_{\mathrm{s}}^{0} \rightarrow \mathrm{J} / \psi \mathrm{K}^{+} \mathrm{K}^{-}$decay. In total, approximately 9000 decay candidates are used to make the measurement, from $5.0 \mathrm{fb}^{-1}$ of data collected between 2011 and 2016 [10]. The number of candidates is much smaller than that used for the measurement of $B_{\mathrm{s}}^{0} \rightarrow \mathrm{J} / \psi \mathrm{K}^{+} \mathrm{K}^{-}$decays, therefore external constraints are taken 
Table 2 Values of the principal physics parameters determined from the polarisation-independent fit. The first uncertainty is statistical and the second systematic.

\begin{tabular}{lc} 
Parameter & Value \\
\hline$\phi_{\mathrm{s}}^{\mathrm{s} s \mathrm{~s}}[\mathrm{rad}]$ & $-0.07 \pm 0.13 \pm 0.03$ \\
$|\lambda|$ & $1.02 \pm 0.05 \pm 0.03$ \\
$\left|A_{0}\right|^{2}$ & $0.382 \pm 0.008 \pm 0.011$ \\
$\left|A_{\perp}\right|^{2}$ & $0.287 \pm 0.008 \pm 0.005$ \\
\hline
\end{tabular}
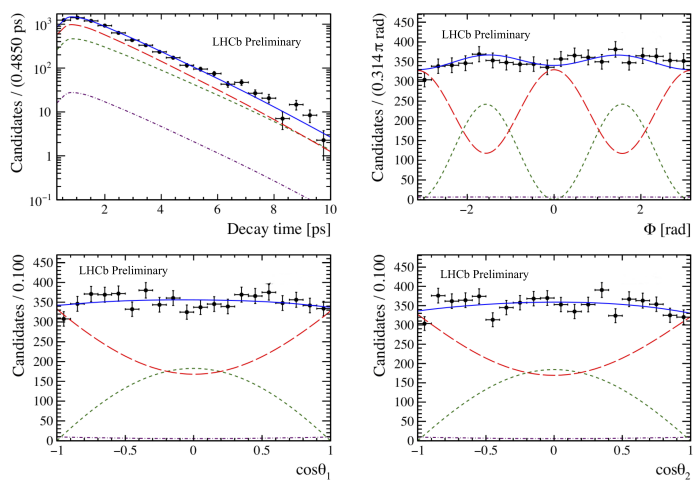

Fig. 2 Decay-time and helicity-angle distributions for $B_{\mathrm{s}}^{0} \rightarrow \phi \phi$ decays (data points) with the onedimensional fit projections overlaid. The solid blue line shows the total signal contribution, which is composed of $C P$-even (long-dashed red), $C P$-odd (short-dashed green) and $\mathrm{S}$-wave (dotted-dashed purple) contributions [10].

from the best known values of $\Delta m_{\mathrm{s}}, \Gamma_{\mathrm{s}}$, and $\Delta \Gamma_{\mathrm{s}}$. The four observables describing the decay are shown in Figure 2 along with the corresponding fit projections. Numerical results for the polarisation amplitudes, $\left|A_{0}\right|^{2}$ and $\left|A_{\perp}\right|^{2}$, are given in Table 2, along with the $C P$-violating parameters, $\phi_{\mathrm{s}}^{\mathrm{s} \overline{\mathrm{s}}}$ and $|\lambda|$. Results are consistent with $C P$ conservation and consequently SM predictions.

\subsection{The $B_{\mathrm{s}}^{0} \rightarrow \mathrm{K}^{+} \pi^{-} \mathrm{K}^{-} \pi^{+}$decay}

A further example of a decay channel that requires an angular analysis is given by the $B_{\mathrm{s}}^{0} \rightarrow \mathrm{K}^{+} \pi^{-} \mathrm{K}^{-} \pi^{+}$decay. The decay mode is further complicated by the many contributions to the $\mathrm{K} \pi$ mass spectrum. These contributions consist of non-resonant $S$-wave, $\mathrm{K}^{* 0} P$-wave, in addition to that of a tensor $\overline{\mathrm{K}}_{2}^{*}(1430)^{0}$ along with associated interferences. In order to properly disentangle so many contributions, the decay-time dependent fit incorporates the $\mathrm{K} \pi$ invariant mass as an observable. The $\mathrm{K} \pi$ invariant mass along with the associated fit is shown in Figure 3. The dataset used for the analysis consists of $3 \mathrm{fb}^{-1}$ of LHCb data collected in 2011 and 2012 [11]. 


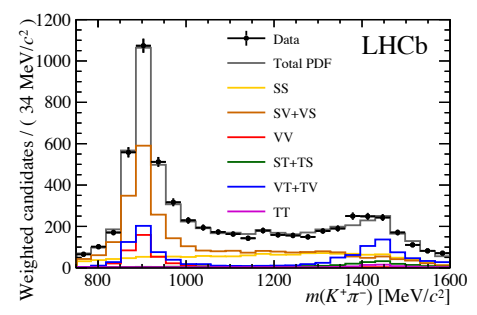

Fig. 3 One-dimensional projections of the decay-time-dependent, flavour-tagged fit to (black points) the sPlot weighted data for the $\mathrm{K}^{+} \pi^{-}$invariant mass. The solid gray line represents the total fit model along with the iCP-averaged components for each contributing decay [11].

The results of the $C P$-violation parameters, $\phi_{\mathrm{s}}^{d \bar{d} \mathrm{~s}}$ and $|\lambda|$, are given in Table 3 . $C P$ violation is consistent with the SM expectation. The analysis is dominated by systematic uncertainties, the largest contribution of which originates from the size of the simulation sample.

Table 3 Values of the $C P$ violation parameters determined from the polarisation-independent fit. The first uncertainty is statistical and the second systematic.

$$
\begin{array}{lc}
\text { Parameter } & \text { Value } \\
\hline \phi_{\mathrm{s}}^{d \bar{d} \mathrm{~s}}[\mathrm{rad}] & -0.10 \pm 0.13 \pm 0.14 \\
|\lambda| & 1.035 \pm 0.034 \pm 0.089 \\
\hline
\end{array}
$$

\section{4 $C P$ violation in $B \rightarrow h h$ decays}

The measurement of $C P$ violation in $B \rightarrow h h$ decays is a measurement of timedependent $C P$ violation in both $B_{\mathrm{s}}^{0}$ and $B^{0}$ decays, as different mesons are the main contributors to different final states. The $\pi \pi$ final state is studied with $B^{0}$ decays and the KK final state with $B_{\mathrm{s}}^{0}$ decays. The $B^{0} \rightarrow \mathrm{K}^{-} \pi^{+}$decay is used to understand the decay time acceptance and calibrate the incorrect flavour assignment probability. The fit to all final states simultaneously allows for mis-identified pions and kaons to be accounted for. The analysis used $3.0 \mathrm{fb}^{-1}$ of data collected in 2011 and 2012 [16].

The results of the $C P$ violation parameters defined in eq. 1 are shown in Table 4. The significance for $\left(C_{K K}, S_{K K}, A_{C P}^{B_{\mathrm{s}}^{0}}\right)$ do deviate from $(0,0,-1)$ has been determined from a $\chi^{2}$ test statistic to be 4.0 standard deviations. This result constitutes the strongest evidence for $C P$ violation in the $B_{\mathrm{s}}^{0}$ system to date. 
Table 4 Values of the principal physics parameters determined from the polarisation-independent fit. The first uncertainty is statistical and the second systematic.

\begin{tabular}{lc} 
Parameter & Value \\
\hline$C_{\pi \pi}$ & $-0.34 \pm 0.06 \pm 0.01$ \\
$S_{\pi \pi}$ & $-0.63 \pm 0.05 \pm 0.01$ \\
$C_{K K}$ & $0.20 \pm 0.06 \pm 0.02$ \\
$S_{K K}$ & $0.18 \pm 0.06 \pm 0.02$ \\
$A_{K K}^{\Delta \Gamma}$ & $-0.79 \pm 0.07 \pm 0.10$ \\
$A_{C P}^{B^{0}}$ & $-0.084 \pm 0.004 \pm 0.003$ \\
$A_{C P}^{B_{\mathrm{s}}^{0}}$ & $0.213 \pm 0.015 \pm 0.007$ \\
\hline
\end{tabular}

\section{Time-dependent $C P$ violation in $B^{0}$ decays}

\subsection{The $B^{0} \rightarrow \mathrm{K}_{S}^{0} \pi^{0} \pi^{0}$ decay}

The known energy of electron-positron collisions results in a fully kinematically constrained decay. This allows the use of the beam-constrained mass, $M_{b c} \equiv$ $\sqrt{\left(E_{\text {beam }} / c^{2}\right)^{2}-\left|\mathbf{p}_{B}^{C M} / c\right|^{2}}$, and the energy difference $\Delta E \equiv E_{\text {beam }}-E_{B}^{C M}$, to isolate the signal candidates from the background candidates, where $\mathbf{p}_{B}^{C M}$ and $E_{B}^{C M}$ are the B momentum and energy respectively in the centre-of-mass (CM) frame. A common variable used in addition to isolate signal is a likelihood ratio calculated from modified Fox-Wolfram moments, $\mathcal{R}_{s / b}[13]$.

The signal and background distributions of $M_{b c}, \Delta E$, and $\mathcal{R}_{s / b}$ are shown in Figure 4, where $335 B^{0} \rightarrow \mathrm{K}_{S}^{0} \pi^{0} \pi^{0}$ signal candidates are seen from a three dimensional fit. The total dataset consists of $742 \times 10^{6} B \bar{B}$ pairs collected with the Belle detector at the $\Upsilon(4 S)$ resonance. The high effective flavour tagging power of $29.8 \pm 0.4 \%$ makes a decay-time dependent fit possible with the number of candidates found. The resulting decay-time dependent fit measures a values of $\mathcal{S}=-0.92_{-0.27}^{+0.31}$ (stat) \pm 0.11 (syst) and $\mathcal{A}=-0.28 \pm 0.21$ (stat) \pm 0.04 (syst).

\subsection{The $B^{0} \rightarrow \mathrm{K}_{S}^{0} \eta \gamma$ decay}

A similar decay time dependent analysis is performed using $B^{0} \rightarrow \mathrm{K}_{S}^{0} \eta \gamma$ decays as that described in the previous section. The total dataset consists of $772 \times 10^{6} B \bar{B}$ pairs collected with the Belle detector at the $\Upsilon(4 S)$ resonance. A total of 92 candidates are used to make the measurement. The $C P$ violation parameters are determined to be $\mathcal{S}=-1.32 \pm 0.77$ (stat) \pm 0.36 (syst) $\mathcal{A}=-0.48 \pm 0.41$ (stat) \pm 0.07 (syst). The 1 , 

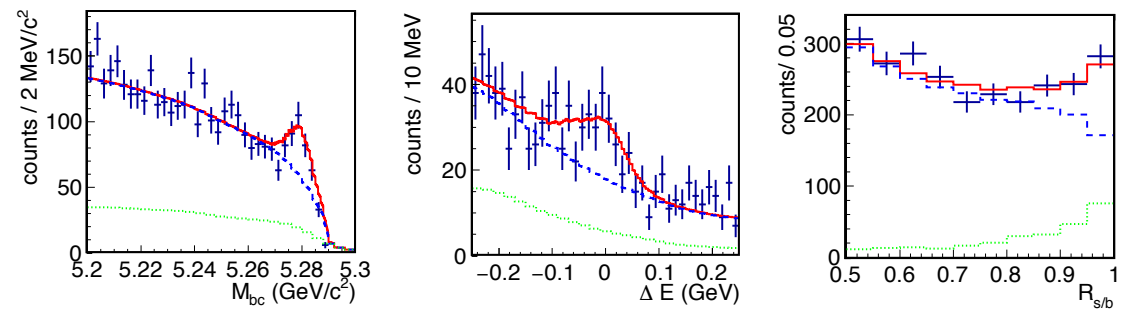

Fig. $4 M_{b c}, \Delta E$ and $\mathcal{R}_{s / b}$ distributions (points with uncertainties) using signal-enhanced selections $M_{b c}>5.27 \mathrm{GeV} / c^{2}, 0.15 \mathrm{GeV}<\Delta E<0.10 \mathrm{GeV}$, and $\mathcal{R}_{s / b}>0.9$ except for the variable displayed. The fit result is illustrated by the solid curve, while the total and $B \bar{B}$ backgrounds are shown by broken and dotted curves, respectively [13].

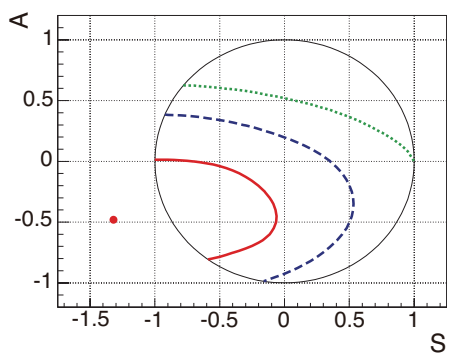

Fig. 5 The solid red, dashed blue, and dotted green curves show the $1 \sigma, 2 \sigma, 3 \sigma$ confidence contours, respectively. The red dot shows the fit result. The physical boundary $S^{2}+A^{2}=1$ is drawn with a thin solid black curve. Our result is consistent with a null asymmetry within $2 \sigma$ [12].

2, and $3 \sigma$ confidence contours of the $\mathcal{S}$ and $\mathcal{A}$ parameters are shown in Figure 5. The result is consistent with null asymmetry within $2 \sigma$.

\subsection{Measurement of $\cos (2 \beta)$ using $B^{0} \rightarrow D^{*} h^{0}$ decays}

The first evidence for the resolution of the sign of $\cos (2 \beta)$ has required the combination of $471 \times 10^{6} B \bar{B}$ pairs collected with the BaBar detector and $772 \times 10^{6} B \bar{B}$ pairs collected with the Belle detector [14]. The measurement of $\cos (2 \beta)$ is achieved through the interference of the $D^{*}$ and $\bar{D}^{*}$ decay amplitudes, $\mathcal{A}_{D^{*}}$ and $\mathcal{A}_{\bar{D}^{*}}$, which can be written as

$$
\operatorname{Im}\left(e^{-i 2 \beta} \mathcal{A}_{D^{*}} \mathcal{A}_{\bar{D}^{*}}\right)=\operatorname{I} m\left(\mathcal{A}_{D^{*}} \mathcal{A}_{\bar{D}^{*}}\right) \cos (2 \beta)-\mathcal{R} e\left(\mathcal{A}_{D^{*}} \mathcal{A}_{\bar{D}^{*}}\right) \sin (2 \beta) .
$$

This then gives access to both $\sin (2 \beta)$ and $\cos (2 \beta)$, which are measured to be $0.80 \pm 0.14 \pm 0.06 \pm 0.03$ and $0.91 \pm 0.22 \pm 0.09 \pm 0.07$, respectively, where the first uncertainty is statistical, the second is systematic and the third is related to the choice 
of fit model. The significance of which $\cos (2 \beta)$ is greater than zero is determined to be $3.7 \sigma$, representing the first evidence.

\section{Summary}

The most accurate single measurement of $C P$ violation in $B_{\mathrm{s}}^{0}$ mixing has been presented along with an updated measurement of the weak phase in the $B_{\mathrm{s}}^{0} \rightarrow \phi \phi$ decay and the first measurement of the weak phase in $B_{\mathrm{S}}^{0} \rightarrow \mathrm{K}^{+} \pi^{-} \mathrm{K}^{-} \pi^{+}$decays using LHCb data. An LHCb analysis has also revealed the strongest evidence yet of $C P$ violation in the $B_{\mathrm{s}}^{0}$ sector, measured from $B_{\mathrm{s}}^{0} \rightarrow \mathrm{K}^{+} \mathrm{K}^{-}$decays to be at the $4.0 \sigma$ level. Two new decay time dependent measurements have been presented using data collected with the Belle detector, $B^{0} \rightarrow \mathrm{K}_{S}^{0} \pi^{0} \pi^{0}$ and $B^{0} \rightarrow \mathrm{K}_{S}^{0} \eta \gamma$. In addition, first evidence has been presented that $\cos (2 \beta)>0$, with a significance of $3.7 \sigma$, potentially resolving the long standing ambiguity in the value of the CKM angle $\beta$.

\section{References}

1. J. Charles, et al., Phys. Rev. D91, 073007 (2015). DOI 10.1103/PhysRevD.91.073007

2. P. Ball, R. Fleischer, Eur. Phys. J. C48, 413 (2006). DOI 10.1140/epjc/s10052-006-0034-4

3. A. Lenz, Phys. Rev. D76, 065006 (2007). DOI 10.1103/PhysRevD.76.065006

4. M. Tanabashi, et al., Phys. Rev. D98(3), 030001 (2018). DOI 10.1103/PhysRevD.98.030001

5. M. Raidal, Phys. Rev. Lett. 89, 231803 (2002). DOI 10.1103/PhysRevLett.89.231803

6. B. Bhattacharya, A. Datta, M. Duraisamy, D. London, Phys.Rev. D88, 016007 (2013). DOI 10.1103/PhysRevD.88.016007

7. M. Bartsch, G. Buchalla, C. Kraus, (2008)

8. H.Y. Cheng, C.K. Chua, Phys.Rev. D80, 114026 (2009). DOI 10.1103/PhysRevD.80.114026

9. R. Aaij, et al., Phys. Rev. Lett. 114(4), 041801 (2015). DOI 10.1103/PhysRevLett.114.041801

10. R. Aaij, et al. Measurement of CP violation in $B_{s}^{0} \rightarrow \phi \phi$ decays (2018)

11. R. Aaij, et al., JHEP 03, 140 (2018). DOI 10.1007/JHEP03(2018) 140

12. H. Nakano, et al., Phys. Rev. D97(9), 092003 (2018). DOI 10.1103/PhysRevD.97.092003

13. Y. Yusa, et al., Submitted to: Phys. Rev. D (2018)

14. I. Adachi, et al., (2018)

15. L. Di Luzio, M. Kirk, A. Lenz, Phys. Rev. D97(9), 095035 (2018). DOI 10.1103/PhysRevD.97.095035

16. R. Aaij, et al., Phys. Rev. D98(3), 032004 (2018). DOI 10.1103/PhysRevD.98.032004 